Conclusion: We found psychiatric symptoms such as obsessive-compulsive besides somatization, anxiety, depression, hostility and sleep disturbances higher in the patients with CSP. Additionally, psychiatric symptoms, anxious and depressive temperaments were more common in the patients with fair/poor shoulder function. Self-esteem was not related with the pain. All these findings indicate the importance of psychological health in the patients with CSP. In order to reach treatment goals, psychiatric symptoms and temperament profiles of these patients also should be considered.

Disclosure of Interests: : None declared

DOI: 10.1136/annrheumdis-2020-eular.3817

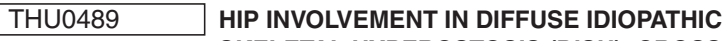 SKELETAL HYPEROSTOSIS (DISH): CROSS- SECTIONAL STUDY}

T. Clavaguera $^{1,2}$, E. Armengol ${ }^{3}$, M. Buxó ${ }^{4}$, M. Valls ${ }^{1,2}$, E. DE Cendra ${ }^{2}$, P. Reyner ${ }^{1,2}{ }^{1}$ Hospital Universitari Docotr Trueta, Rheumatology, Girona, Spain; ${ }^{2}$ Hospital Santa Caterina, Rheumatology, Salt, Spain; ${ }^{3}$ Hospital de Palamos, Rheumatology, Palamós, Spain; ${ }^{4}$ Institut d'Investigació Biomèdica de Girona, Biostatistics, Girona, Spain

Background: In DISH or Forestier-Rotés disease, hip involvement is often misdiagnosed as hip osteoarthritis, especially when it is the initial manifestation of the disease or in patients with scarce vertebral signs. At present, a correct identification of this entity may suppose considerable therapeutic implications ${ }^{1}$.

Objectives: The purpose of this study was to assess the prevalence and characteristics of hip involvement in our cohort of patients with DISH and evaluate the association of this extra-spinal manifestation with the variables studied.

Methods: We carried out a cross-sectional study in DISH patients who met Resnick and / or Utsinger classification criteria. We collected demographic, anthropometric, clinical and imaging data. Hip involvement was defined as the characteristic irregular bony excrescences above acetabulum. The cohort was divided between patients with and without hip involvement. A univariate descriptive analysis was performed with means and standard deviations, absolute frequencies and percentages. The normality of the data was checked using the Shapiro-Willis test. The bivariate analysis, for the qualitative variables, the $x^{2}$ test or Fisher's exact test were identified. For the quantitative variables, the Student's t-test was used if the data followed a normal distribution, and otherwise using the Mann-Whitney $U$ test.

Results: Of the 58 patients included, $67.2 \%$ were male. The median age was 69.4 years (44-89). The average time of disease evolution was $14.8(+9.3)$ years. Although the most frequent initial symptom was thoraco-lumbar pain (39.7\%), hip complaints were initially present in $13.8 \% .22,6 \%$ of patients did not fulfil Resnick classification criteria. Hip involvement was identified in $53.4 \%$ and a $61,3 \%$ the cartilage space were preserved. In the bivariate analysis, hip involvement was associated with female sex, a reduction in the intermaleolar distance (IM) and the presence of certain enthesopathies (greater trochanter, superior iliac spines and distal patellar entheses). The acetabular ossifications were significantly related to the mixed pattern compared to the other possible phenotypes of the disease.

Conclusion: Hip involvement has been described in more than $50 \%$ of our patients. We found out that it was associated with female sex and a more broad ossification phenotype (mixed pattern). The measurement of IM distance could be useful for the clinical evaluation of this condition. Ossifications of other pelvic ring entheses were more observed in association with acetabular hyperostosis than other peripheral insertions.

References:

[1] Jung-Mo Hwang, Deuk-Soo Hwang, Chan Kang, Woo-Yong Lee, et al. Arthroscopic Treatment for Femoroacetabular Impingement with Extraspinal Diffuse Idiopathic Skeletal Hyperostosis. Clin Orthop Surg 2019; 11: 275-281.

Disclosure of Interests: : Teresa Clavaguera Speakers bureau: novartis, BMS, Faes, Eulàlia Armengol Speakers bureau: Novartis, Maria Buxó: None declared, Marta Valls: None declared, Eulàlia DE CENDRA: None declared, Patricia Reyner Speakers bureau: Faes, Sanofi

DOI: 10.1136/annrheumdis-2020-eular.2590

\section{THU0490 \\ DEEP FRICTION MASSAGE VERSUS LOCAL STEROID INJECTION FOR TREATMENT OF PLANTAR FASCIITIS: A RANDOMIZED CONTROLLED TRIAL}

A. Moshrif ${ }^{1}$, M. Elwan ${ }^{1}$, U. Daifallah ${ }^{2} .{ }^{1}$ Al Azhar University, Assiut, Egypt; ${ }^{2}$ Sohag University, Sohag, Egypt

Background: Deep friction massage (DFM) has long been proven to be effective in the treatment of some tendinopathies.

Objectives: to evaluate the efficacy of DFM in the treatment of plantar fasciitis (PF) in comparison with local steroid injection.

Methods: In this randomized controlled trial, 60 patients with PF were selected from those attending the rheumatology and rehabilitation outpatient clinic and then randomized to receive either $40 \mathrm{mg}$ triamcinolone local injection (group I: 30 patients; 41 heals) using the medial approach or to receive seven sessions of deep transverse friction massage (10 min each) every other day (group II: 30 patients; 36 heels). The outcome measures were the pain and function assessment by visual analog scale and foot function index, respectively, at 2 and 6 weeks of follow-up. Results: Demographic data showed a statistically insignificant difference in age, female to male ratio, and BMI in both groups. The mean age was 39.42 years in group I and 41.32 years in group II $(P=0.86)$. The female to male ratio was $3: 1$ in group I and 2.75: 1 in group II, and the mean BMI was 32.41 in group I and 33.31 in group II $(\mathrm{P}=0.51)$. At 2 and 6 weeks of follow-up, DFM led to less improvement in pain and function compared with local steroid injection $(P=0.001$ and 0.002 , respectively, at both time points of follow-up).

Conclusion: This study revealed that DFM is not effective as a single modality in treatment of PF. Further large-scale studies are needed to support this observation.

Disclosure of Interests: : None declared

DOI: 10.1136/annrheumdis-2020-eular.5402

\section{THU0491 HYALRONIC ACID INJECTION IN PLANTER FASCIITIS: CAN WE USE?}

A. Zakria ${ }^{1}$, A. Hafez ${ }^{2}$, A. Abdel-Nasser ${ }^{3} .{ }^{1}$ Minia University Hospital, Minia, Egypt; ${ }^{2}$ Minia University Hospital, Rheumatology, Minia, Egypt; ${ }^{3}$ Minia University, Minia, Egypt

Background: Plantar fasciitis is the most common cause of hind foot pain. Treatment is aimed at pain reduction and maintaining or improving function. Several types of injected materials are used including corticosteroids, hyaluronic acid and Platelet-rich plasma.

Objectives: The aim of this study was to evaluate the efficacy of hyaluronic acid injection in comparison to corticosteroid injection for the treatment of degenerative plantar fasciitis.

Methods: This study included forty patients with degenerative plantar fasciitis diagnosed according to clinical examination and ultrasound findings. They were divided into two groups. Group one included twenty patients who were injected with Methylprednisolone (MP). Group two included another twenty patients who were injected with hyaluronic acid (HA). Clinical assessment was done including morning hee pain, localized heel tenderness, patient assessment of heel pain using the numerical rating scale (NRS), Functional assessment using the Manchester-Oxford foot questionnaire (MOXFQ) and musculoskeletal ultrasound assessment including planter fascia thickness, echogenicity, power Doppler signals, erosions and calcification. Patients were injected and a follow up was done at two and six weeks for each group.

Results: The two groups MP \&HA showed no significant baseline differences in clinical and sonographic variables, while they both significantly improved from baseline after two \&six weeks injection $(\mathrm{P}<0.001)$. The MP group achieved a sig nificant improvement in NRS, MOXFQ, plantar fascial thickness and echogenicity in the first follow up after two weeks better than the second follow up afte six weeks $(P<0.001)$. The HA group achieved significant improvement in NRS MOXFQ, plantar fascial thickness and echogenicity in the second follow up after six weeks better than the first follow up after two weeks $(P<0.001)$.

Over all, MP group had significant improvement clinically and sonographically than HA group after two weeks $(\mathrm{P}<0.001)$, while $\mathrm{HA}$ group had significant improvement clinically and sonographically in comparison with MP group after six weeks.

Conclusion: Our study supports the use of a single injection of hyaluronic acid as therapy for plantar fasciitis in preference to methylprednisolone acetate injections, while MP offers short term improvement (after two weeks). HA injection offers better therapeutic advantages in the intermediate-term follow up (after six weeks). Moreover, it has a longer duration of action and enables better tissue healing and can therefore be used as an effective alternative treatment for plantar fasciitis.

Disclosure of Interests: : None declared

DOI: 10.1136/annrheumdis-2020-eular.3037

\section{THU0492 \\ MAGNETIC RESONANCE IMAGING OF THE SACROILIAC JOINTS IN PATIENTS WITH HYPERMOBILITY: A RETROSPECTIVE COHORT STUDY}

A. Jones ${ }^{1}$, C. Ciurtin ${ }^{2}$, H. Kazkaz ${ }^{2}$, M. Hall-Craggs ${ }^{3} .{ }^{1}$ Royal Surrey County Hospital, Guildford, United Kingdom; ${ }^{2}$ University College Hospital, London, United Kingdom; ${ }^{3}$ University College London, London, United Kingdom

Background: The incidence of inflammatory and structural lesions on magnetic resonance imaging of sacroiliac joints (MRI SIJs) in patients with hypermobility related disorders has not been fully investigated. Hypermobile patients are more susceptible to pelvic instability and biomechanical stress of the SIJs, leading to MRI SIJ changes 
similar to those occurring in spondyloarthritis (SpA). Patients with hypermobility and suspected SpA pose a unique challenge owing to the high prevalence of back pain in the hypermobility cohort and the absence of spinal restriction on clinical examination. Objectives: In this study, we aim to investigate the incidence of MRI SIJ lesions in patients with hypermobility.

Methods: We performed a retrospective study of all patients with a confirmed diagnosis of hypermobility related disorders (including hypermobility syndrome, hypermobility spectrum disorders and Ehlers-Danlos Syndromes) referred for an MRI lumbar spine and SIJ between 2011 and 2019 to investigate long-standing back pain. MRIs were examined by a musculoskeletal (MSK) radiologist with more than 25 years of experience, who was blinded to the clinical outcome of the patients. MRI SIJs were assessed for the presence of bone marrow oedema, subchondral sclerosis, erosion, fatty change, enthesitis, ankylosis, joint fluid and capsulitis.

Results: 51 patients with confirmed hypermobility related disorders were referred for MRI SIJ and lumbar spine between 2011 and 2019. 3 patients demonstrated clinical features in keeping with a diagnosis of SpA and were excluded from the study. 15/48 (31.3\%) of patients with hypermobility and back pain (but no clinical picture of SpA) were found to have inflammatory and/or structural lesions on MRI SIJ. The most frequent lesions were small foci of bone marrow oedema (16.6\%) followed by subchondral sclerosis (12.5\%) and fatty change (10.4\%). The incidence of erosions was $4.2 \%$. Conclusion: There is a relatively high incidence of inflammatory and structural lesions on MRI SIJ of patients with hypermobility. The presence of hypermobility should be taken into consideration when interpreting MRI changes in patients with suspected SpA. Further research into long-term outcomes of MRI SIJs in patients with hypermobility and back pain is required to establish the clinical significance of these findings.

Disclosure of Interests: : Alexis Jones: None declared, Coziana Ciurtin Grant/ research support from: Pfizer, Consultant of: Roche, Modern Biosciences, Hanadi Kazkaz: None declared, Margaret Hall-Craggs: None declared DOI: 10.1136/annrheumdis-2020-eular.5686

\section{THU0493 IMPACT OF AGE, GENDER AND EDUCATION LEVEL ON THE CHOICE OF TREATMENT METHOD FOR BACK PAIN AMONG PEOPLE OVER 50 YEARS OF AGE}

M. Nejman ${ }^{1}$, M. Hiller ${ }^{1}$, J. Napora ${ }^{1}$, A. Wojteczek ${ }^{2}$, M. Ziętkiewicz ${ }^{2}$, Z. Zdrojewski ${ }^{2}{ }^{1}$ Students Scientific Circle of Clinical Rheumatology, Department of Internal Medicine, Connective Tissue Diseases and Geriatrics, Medical University of Gdansk, Gdansk, Poland; ${ }^{2}$ Departament of Internal Medicine, Connective Tissue Diseases and Geriatrics, Medical University of Gdansk, Poland, Gdansk, Poland

Background: According to data collected by the Statistics Poland in 2014, lower back pain is the second most common complaint reported by people over the age of 60 and occurs in about $40 \%$ of them. Another $29 \%$ of respondents suffer from pain in other spine sections. Treatment of such a common condition can be a challenge due to the multitude of causes of pain, accompanying diseases and the patient's approach to his own health.

Objectives: The aim of the study was to investigate whether factors such as gender, age, level of education and pain severity affect self-chosen methods of treating back pain

Methods: A survey was conducted on students of Pomeranian Universities Of The Third Age. There were collected socio-demographic characteristics of the participants, features of back pain and detailed information on analgesic methods, including drugs, ointments, exercises and physiotherapy treatments (laser, cryotherapy, hydromassage, ultrasound, heat treatments and other). The responses of participants over the age of 50 were included in the analysis. The answers were divided into groups by age (younger - 50-69 years and older - over 70 years), sex, level of education (lower, medium and higher) and intensity of pain assessed on the VAS scale $(<6$ and $\geq 6)$. The collected data were compared in these groups.

Results: 546 answers were received. 291 respondents were $50-69$ years old, 255 aged $70-90$ and more, $86 \%$ (471) of participants were women. $43 \%$ of respondents had secondary education and the same number had higher education. Over $90 \%$ (494) declared that they have suffered from back pain. Most of them described pain as chronic (56\%), the median pain intensity assessed on the VAS scale was 6 [1-10], and the mean pain intensity was $5.89 \pm 1.79$. $82.6 \%$ of respondents declared doing physical exercises to relieve back pain, $75.9 \%$ were using physiotherapy, $60.7 \%$ were taking analgesic drugs and $54.3 \%$ were using ointments, gels, patches and other local analgesic methods. The higher pain severity was observed in group of females (5.74 vs. $5.61, p=0.002$ ) and people with lower education level (6.52) than in other groups ( 5.89 and $5.68, p=0.005)$. Analgesic drugs were taken more often by younger people $(66.9 \%$ vs. $53.7 \%, p=0.003)$, women $(62.5 \%$ vs. $49.2 \%, p=0.042)$, people of lower education level group ( $80.6 \%$ vs. $62.2 \%$ secondary education group vs. $52.5 \%$ higher education group, $\mathrm{p}<0.001)$ and by people with pain severity $\geq 6(81.5 \%$ vs. $68.9 \%, p<0.001)$. Analgesic gels, ointments, patches and other pharmaceuticals were used more often only in group with higher pain severity $(62.9 \%$ vs. $43.4 \%, p<0.001)$. Performing exercises to relieve pain was more often declared by women $(84.4 \%$ vs. $70.8 \%, p=0.003)$ and people with higher education ( $85.1 \%$ vs. $81.3 \%$ secondary education group vs. $79.1 \%$ lower education group, $p<0.001)$. The study showed that only in groups with varying intensity of pain there was a significant difference in the frequency of using physiotherapy treatments $-68.9 \%$ in group with pain severity $<6$ vs. $81.5 \%$ with pain severity $\geq 6(p<0.001)$.

Conclusion: 1. The severity of pain has the greatest impact on the choice of back pain relief method, but this is not the only important factor.

2. People with lower levels of education and men less often perform physical exercises for treatment regardless of the severity of back pain

3. In the treatment of back pain, attention should be given to recommending the patient an appropriate analgesic method, which will be easily used and more effective.

References:

Population aged 60+. Demographic structure and health. 2016. Statistics Poland Disclosure of Interests: : None declared

DOI: 10.1136/annrheumdis-2020-eular.2883

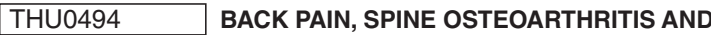 'CANDIDATE GENES' POLYMORPHISM}

N. Pravdyuk ${ }^{1}$, N. Shvyreva ${ }^{1}$, N. Shostak ${ }^{1}$, A. Novikova ${ }^{1}$, E. Zakharova ${ }^{2}$. ${ }^{1}$ Russian National Medical Research University named after N.I. Pirogov, Moscow, Russian Federation; ${ }^{2}$ Medical Genetic Research Center of the Russian Academy of Medical Sciences, Laboratory of Hereditary Metabolic Diseases, Moscow, Russian Federation

Background: Low back pain (LBP) and spine osteoarthritis are among the leading health-related causes of disability and reduction in patient quality of life. More recent work suggested that the factors that lead to disc degeneration may have important genetic components. Genetic predisposition has been confirmed by recent findings of associations between degeneration and gene polymorphisms of matrix macromolecules.

Objectives: Evaluation of genetic polymorphisms of genes collagen $1 \mathrm{~A} 1$ (COL1A1), interleikin $1 \beta$ (IL-1 $\beta$ ), interleikin 6 (IL-6), vitamin D receptor (VDR) in patients with LBP, associated with spine osteoarthritis.

Methods: We examined 33 patients (men-17, women-16, middle age $28,7 \pm 3,77$ ) with LBP end onset, verified by magnetic resonance imaging (MRI) and 15 controls was carried out. Assessed the intensity of pain syndrome on visual analog scale (VAS, $\mathrm{mm}$ ) and the nature of its current (IASP, 1994). Polymerase chain reaction and restriction fragments length polymorphism was used to detect the polymorphism of COL1A1 (rs 1800012), IL-1 (rs 1143627), IL-6 (rs 1800795), VDR (rs 1544410).

Results: The severity of the pain (VAS) average was $60 \mathrm{~mm}$, all the patients revealed chronic option currents pain syndrome. In $86 \%$ of patients detected changes disc in the form of protrusions and hernias, $17 \%$ of patients had a characteristic reducing the height of the intervertebral disc of lower height disc and osteophytes, arthritis facet joints identified in $20 \%$ of patients in $34 \%$ of patients diagnosed changes type Modic II. In $83 \%$ of patients with LBP identified homozygous variant allele (GG) Col1A1 gene. The absence of T-alleles in the gene IL $1 \beta$ in the group of patients was associated with severity of the disease. Carriage of allele in heterozygous AG found in $54 \%$ of patients of the main group and was not observed in the control that requires further accumulation of facts. Identified association GG-genotype of IL-6 clinical and instrumental signs of the syndrome Modic-II.

Conclusion: These findings may be the reason for the patient-specific approach to diagnosing and treatment of back pain. It proves the necessity of research of genetic polymorphisms in patients with spine osteoarthritis.

Disclosure of Interests: : None declared

DOI: 10.1136/annrheumdis-2020-eular.6407

THURSDAY, 04 JUNE 2020

\section{Basic and translational science in paediatric rheumatology}

\begin{tabular}{l|l}
\hline THU0495 & NOVEL UNDERSTANDING OF THE PATHOGENESIS \\
OF JUVENILE IDIOPATHIC ARTHRITIS: FOCUS \\
ON MESENCHYMAL STEM CELLS IMPAIRMENT, \\
SENESCENCE AND IMMUNOREGULATORY FUNCTION
\end{tabular}

L. Zaripova ${ }^{1}$, A. Midgley ${ }^{2}$, S. Christmas ${ }^{3}$, E. Baildam ${ }^{4}$, R. Oldershaw ${ }^{1}$.

${ }^{1}$ University of Liverpool, Musculoskeletal Biology, Liverpool, United Kingdom;

${ }^{2}$ University of Liverpool, Women's Health, Liverpool, United Kingdom;

${ }^{3}$ University of Liverpool, Clinical Infection, Microbiology and Immunology, 